\title{
Discovery of selective inhibitor leads by targeting an allosteric site in Insulin-Regulated Aminopeptidase (IRAP)
}

\author{
Ioannis Temponeras ${ }^{1,3}$, Lykourgos Chiniadis ${ }^{2,3}$, Athanasios Papakyriakou ${ }^{3}$ and Efstratios Stratikos ${ }^{3}$
}

${ }^{1}$ Department of Pharmacy, University of Patras, Greece. ${ }^{2}$ Department of Biotechnology, Agricultural University of Athens, Greece

${ }^{3}$ National Centre for Scientific Research “Demokritos", Agia Paraskevi 15341, Athens, Greece

\section{The scope}

Insulin-regulated aminopeptidase (IRAP) is a transmembrane, zinc-dependent aminopeptidase that performs a variety of important biological functions. IRAP, also known as oxytocinase, leucyl-cystinyl aminopeptidase and placental leucine aminopeptidase is implicated in the regulation of trafficking of glucose transporter type 4, the control of oxytocin levels in pregnancy, as well as the generation of antigenic peptides for cross-presentation. IRAP is also a specific binding site for angiotensin IV, which upon binding serves as a competitive inhibitor of the enzyme. https://www.frontiersin.org/research-topics/12216/physiological-pathological-roles-and-pharmacology-of-insulin-regulated-aminopeptidase IRAP (EC 3.4.11.3) belongs to the M1 family of aminopeptidases and shares high sequence and structural homology with the endoplasmic reticulum aminopeptidases ERAP1 and ERAP2. These enzymes have been shown to adopt conformations between "open" and "closed" states, which expose a large internal cavity for peptide entrance and mediate efficient substrate processing. https://www.frontiersin.org/articles/10.3389/fimmu.2017.00946/full

Due to its involvement in several human disease states, IRAP is an emerging pharmaceutical target. With the aim to discover inhibitors of IRAP that do not disrupt the physiological function of other M1 enzymes, we pursuit the discovery of lead-like compounds that bind away from the highly homologous active site.

\section{The idea}

A recent, high-resolution crystal structure of "closed" ERAP1 in complex with a potent phosphinic pseudopeptide inhibitor revealed distinct druggable pockets occupied by two molecules from the crystallization medium. PDB ID: $6 Q 4 R$

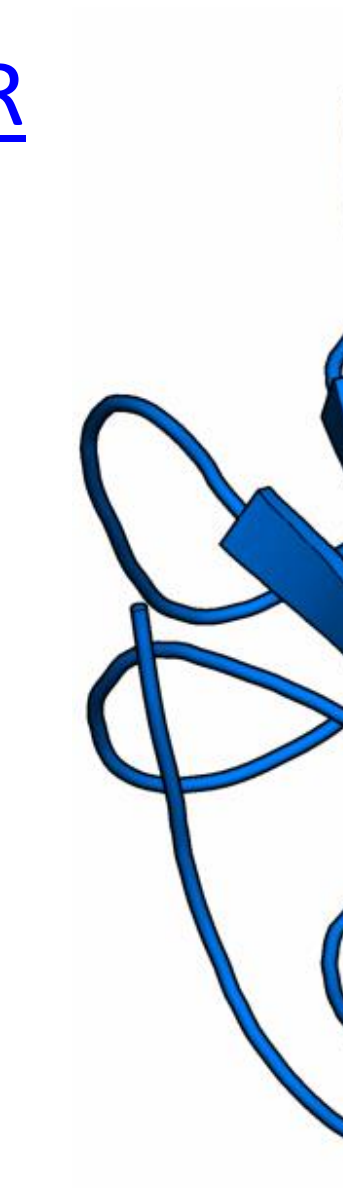

The volume of the substrate cavity is: 2,900 $\AA^{3}$ for ERAP1 and 5,300 $\AA^{3}$ for IRAP consistent with IRAP's physiological role in processing cyclic peptides (oxytocin)

Hinge domain

Pocket 1 (Bis-tris propane) N-terminus Catalytic Zn(II) Active-site bound transition state

\section{e} ( .
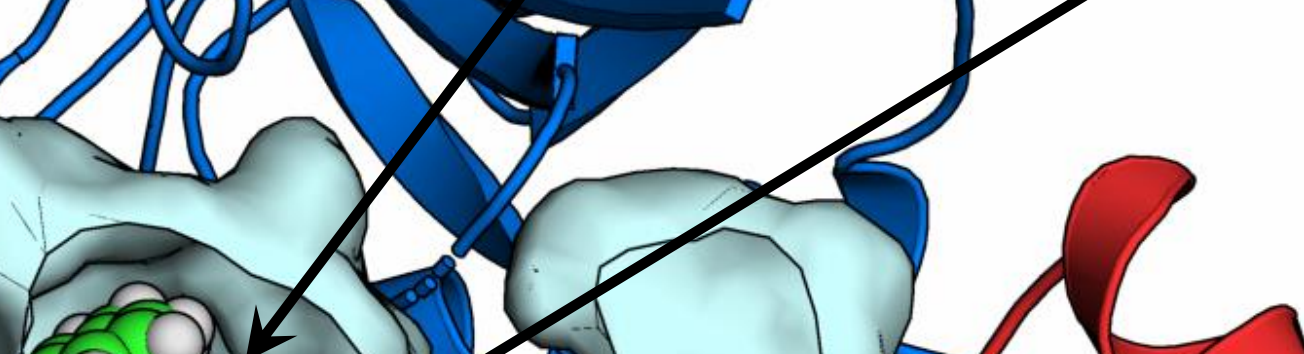

\section{.}
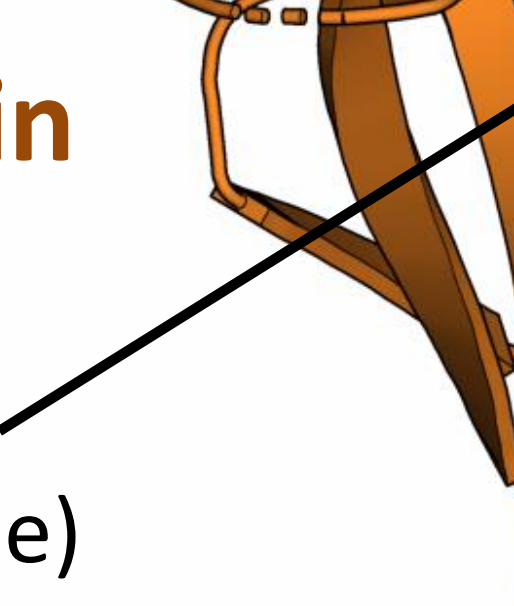

.
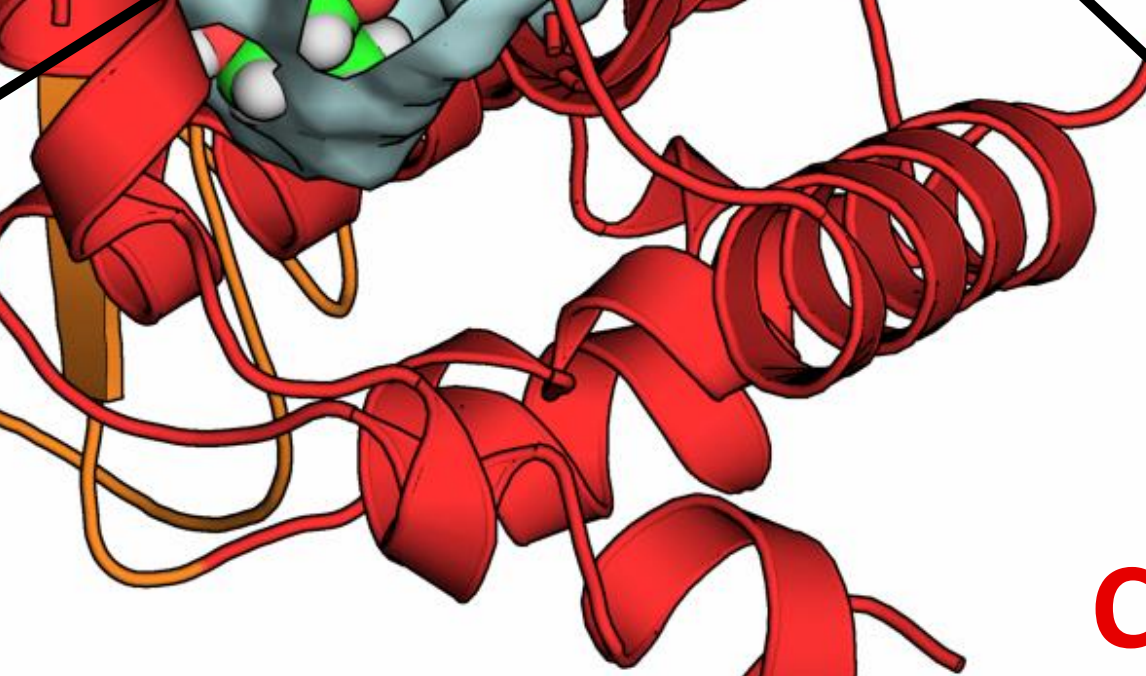

Pocket 2

(malic acid)

C-terminus

We opted to target pocket 1 as the largest pocket and a site that comprise residues

from all three functional domains of the enzymes, next to the hinge domain

\section{Open state}

Substrate receptive
Closed state Catalytically active

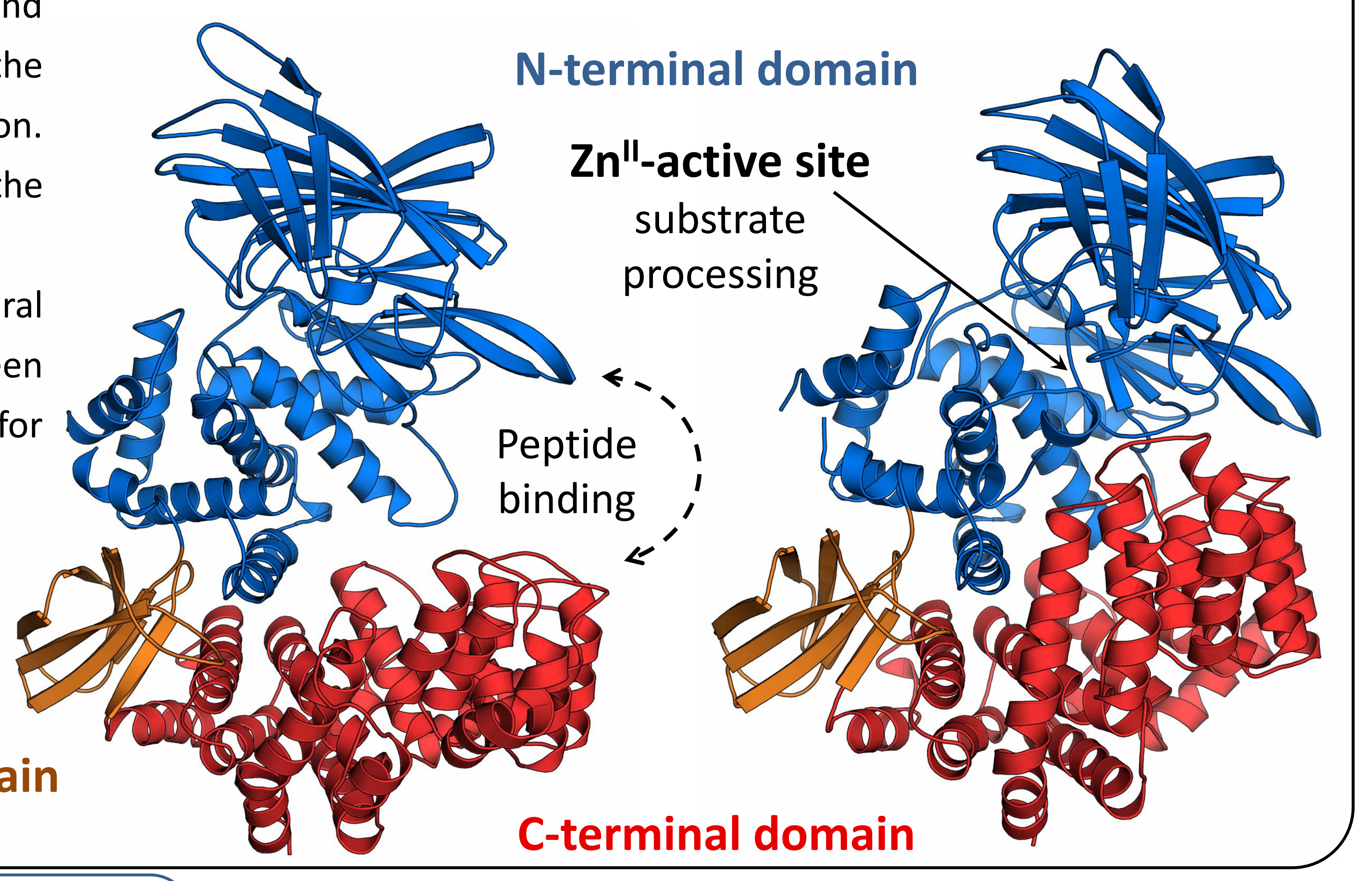

Hinge domain

\section{The method}

First we identified the corresponding pockets in the C-termial domain of IRAP (PDB ID: 5MJ6), which interestingly, share comparably low homology with those of ERAP1 and ERAP2. To discover high-affinity compounds that bind and disrupt the physiological processing of large substrates of IRAP, we employed structure-based virtual screening according to the following steps:

From the purchasable chemical space of the ZINC-15 database, we selected a subset of lead-like compounds (MW of 200-350 and cLogP < 3.0), clean from reactive groups or PAINS (anodyne subset) and in-stock. A total of 2.6 million compounds were docked at the selected site of IRAP using AutoDock VINA in order to select the top-ranked $1 \%$ compounds with the highest affinity.

These compounds were also docked at the related pocket of ERAP1, so as to disfavor compounds of equally high affinity for the enzyme with the highest homology to IRAP. After visual investigation of residue-specific interactions with the topranked compounds, we selected 305 compounds for molecular dynamics simulations of 20 ns each ( $>6 \mu$ s in total). From the second half of the MDs we obtained 1,000 snapshots and carried out free energy calculations with the MM/GBSA and MM/PBSA methods, which guided the final selection of the most promising compounds for experimental investigation.

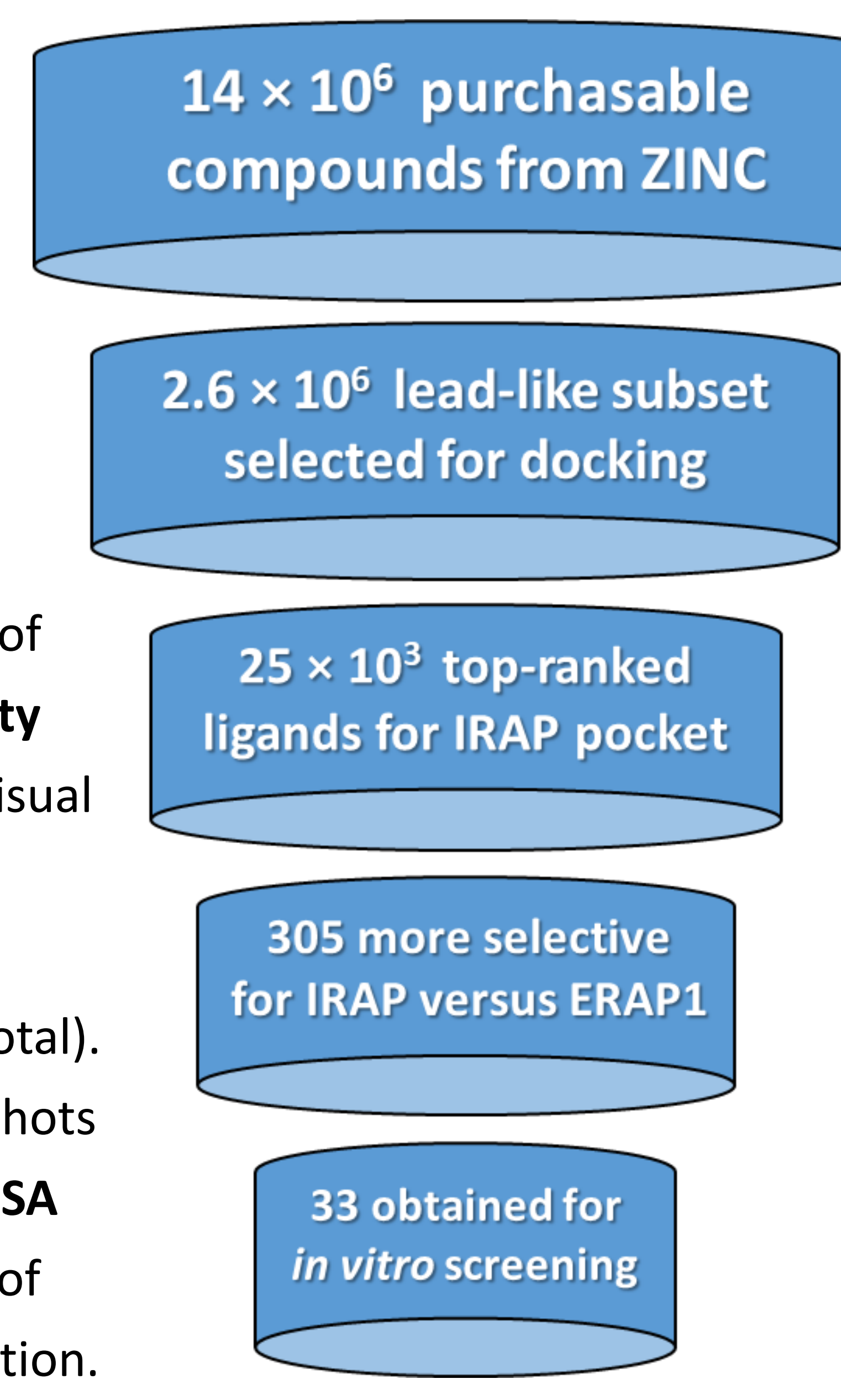

For their evaluation we employed two orthogonal functional assays: one using a small fluorigenic substrate (Leu-AMC), and one by following the degradation of oxytocin by IRAP using HPLC.

\section{The result}

Screening of the compounds using the fluorigenic Leu-AMC substrate revealed 3 compounds with $\mathrm{IC}_{50}$ values $<100 \mu \mathrm{M}$ and characteristic inhibition plots shown.

\begin{tabular}{|c|c|c|c|c|c|}
\hline ID & $\begin{array}{c}\mathrm{IC}_{50} \\
(\mu \mathrm{M})\end{array}$ & ID & $\begin{array}{c}\text { I } \\
(\mu \mathrm{M})\end{array}$ & ID & $\begin{array}{c}\mathrm{IC}_{50} \\
(\mu \mathrm{M})\end{array}$ \\
\hline 1 & $\mathrm{NI}$ & 12 & $\mathrm{NI}$ & 23 & 333 \\
\hline 2 & $\mathrm{NI}$ & 13 & 192 & 24 & 337 \\
\hline 3 & 192 & 14 & $\mathrm{NI}$ & 25 & $\mathrm{NI}$ \\
\hline 4 & $\mathrm{NI}$ & 15 & $\mathrm{NI}$ & 26 & $\mathbf{N I}$ \\
\hline 5 & 76 & 16 & $\mathrm{NI}$ & 27 & $\mathrm{NI}$ \\
\hline 6 & $\mathrm{NI}$ & 17 & $\mathbf{5 6}$ & 28 & $\mathbf{9 2}$ \\
\hline 7 & $\mathrm{NI}$ & 18 & $\mathrm{NI}$ & 29 & $\mathrm{NI}$ \\
\hline 8 & $\mathrm{NI}$ & 19 & $\mathrm{NI}$ & 30 & $\mathrm{NI}$ \\
\hline 9 & $\mathrm{NI}$ & 20 & 584 & 31 & 193 \\
\hline 10 & $\mathrm{NI}$ & 21 & $\mathrm{NI}$ & 32 & $\mathrm{NI}$ \\
\hline 11 & $\mathrm{NI}$ & 22 & $\mathrm{NI}$ & 33 & $\mathrm{NI}$ \\
\hline
\end{tabular}

NI: no inhibition up to $1 \mathrm{mM}$ compound
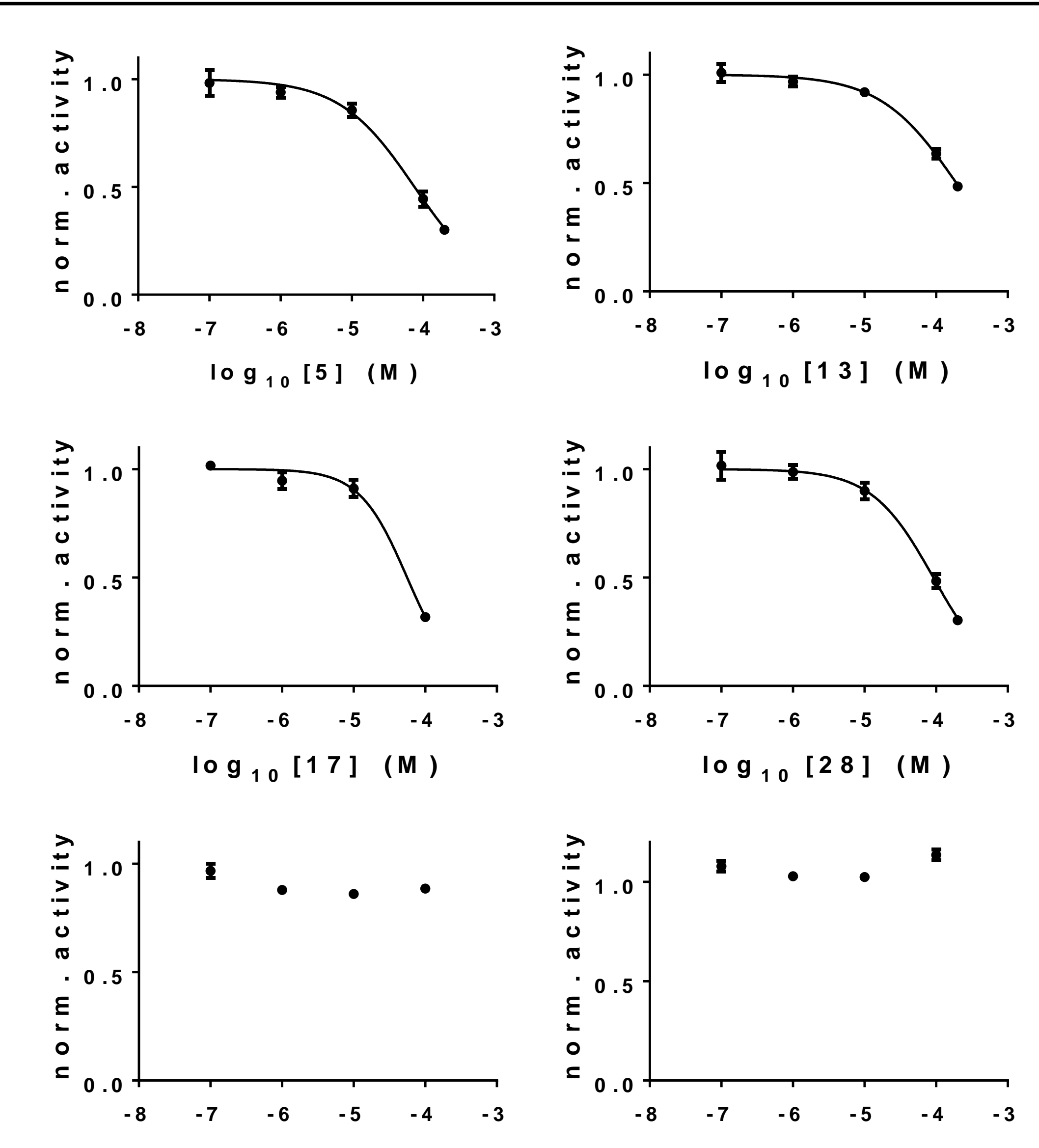

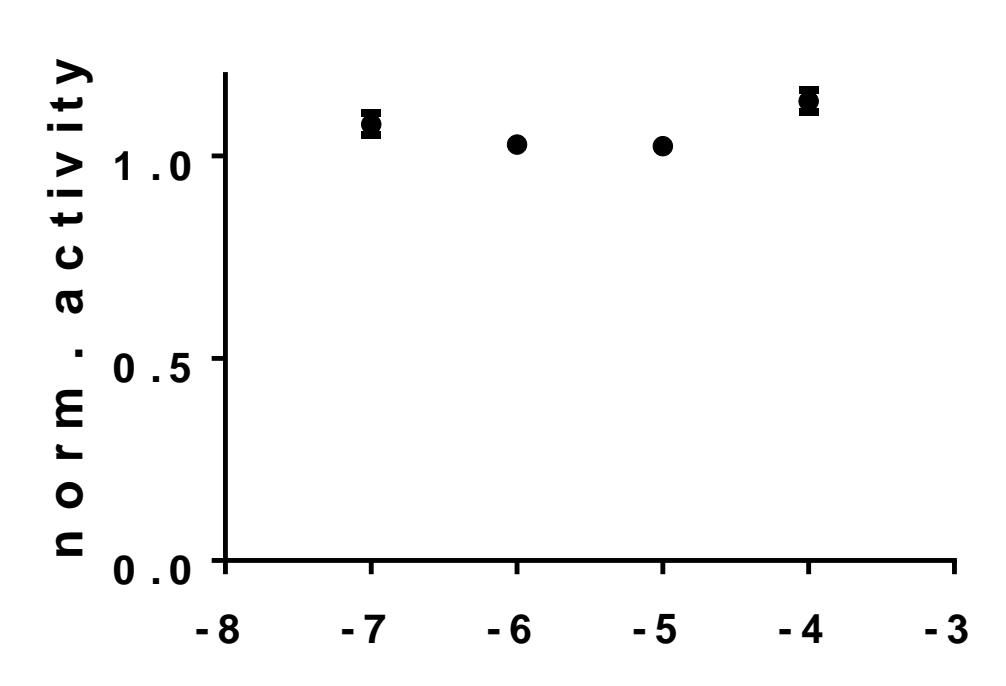

Interestingly, compounds that inhibit Leu-AMC with $\mathrm{IC}_{50}<100 \mu \mathrm{M}$ did not display similar inhibition of the natural substrate, oxytocin. In contrast, compound 26 that does not inhibit Leu-AMC, exhibited $69 \%$ inhibition of the cyclic substrate at $100 \mu \mathrm{M}$ tested. From the dose-dependent inhibition of oxytocin degradation by IRAP, followed by HPLC in the presence of compound $\mathbf{2 6}$, we estimated an $\mathbf{I C}_{\mathbf{5 0}}$ value of $\mathbf{4 0} \boldsymbol{\mu M}$ (shown below).

\begin{tabular}{|c|c|c|c|c|c|}
\hline ID & $\begin{array}{c}\% \\
\text { Inhib. }\end{array}$ & ID & $\begin{array}{c}\% \\
\text { Inhib. }\end{array}$ & ID & $\begin{array}{c}\% \\
\text { Inhib. }\end{array}$ \\
\hline 1 & 0 & 12 & 44 & 23 & 26 \\
\hline 2 & 26 & 13 & 10 & 24 & 0 \\
\hline 3 & 7 & 14 & 25 & 25 & 14 \\
\hline 4 & 11 & 15 & 9 & 26 & 69 \\
\hline 5 & 17 & 16 & 32 & 27 & 32 \\
\hline 6 & 15 & 17 & 23 & 28 & 0 \\
\hline 7 & 0 & 18 & 0 & 29 & 39 \\
\hline 8 & 0 & 19 & 0 & 30 & 22 \\
\hline 9 & 30 & 20 & 34 & 31 & 10 \\
\hline 10 & 0 & 21 & 14 & 32 & 28 \\
\hline 11 & 13 & 22 & 0 & 33 & 10 \\
\hline
\end{tabular}

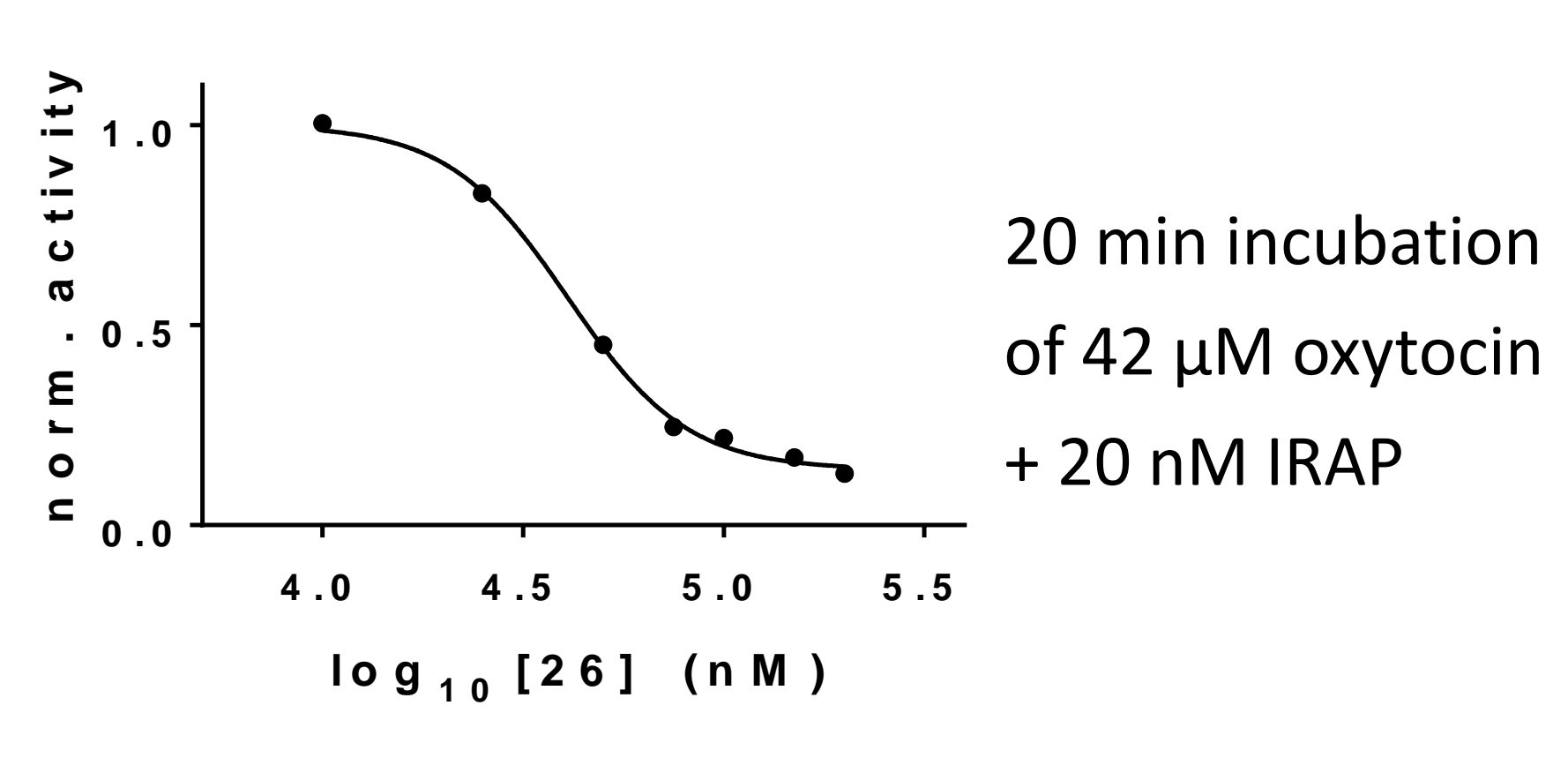

Lead compound 26 exhibits a size-specific substrate inhibition profile for IRAP,

suggesting that targeting a C-domain pocket away from the active site is a viable strategy to develop selective inhibitors. 